\title{
Mortalidad perinatal en el parto prematuro entre 22 y 34 semanas en un hospital público de Santiago, Chile
}

\author{
Alfredo Ovalle $S .{ }^{1}$, Elena Kakarieka $W^{2}{ }^{2}$, Marcela Díaz $C .^{1}$, Trinidad García Huidobro \\ M. ${ }^{1}$, María Jesús Acuña M. ${ }^{1}$, Carla Morong $C^{2}{ }^{2}$, Selim Abara C. ${ }^{a}$, Ariel Fuentes G. $^{1}$
}

${ }^{1}$ Servicio y Departamento de Obstetricia, Ginecología y Neonatología; ${ }^{2}$ Servicio de Anatomía Patológica. Hospital San Borja Arriarán. Facultad de Medicina, Universidad de Chile.

a Alumno, Escuela de Medicina, Universidad de Chile.

\section{RESUMEN}

Objetivo: Conocer la mortalidad perinatal del parto prematuro y los factores de riesgo asociados, usando los datos perinatales clínicos, los resultados de laboratorio y los hallazgos patológicos del feto, neonato y placenta. Métodos: Estudio retrospectivo, cohorte de 407 nacimientos prematuros, únicos, entre 22.0 y 34.0 semanas de gestación. Se estudiaron las muertes fetales y neonatales hasta los 7 días de vida. Fallecieron 122 niños (64 muertes fetales y 58 neonatales), 78 tuvieron autopsia. Resultados: La mortalidad perinatal fue de $30 \%(122 / 407)$. El $71 \%(87 / 122)$ de las muertes ocurrieron antes de las 30 semanas y el $81 \%$ (99/122) en nacidos con peso menor de 1500 gramos. Las principales causas de muerte perinatal según el factor asociado con el parto prematuro fueron: infección bacteriana ascendente (IBA) 41\% (50/122), anomalía congénita 20\% (24/122) e hipertensión arterial 12\% (15/122). Los factores de riesgo de muerte perinatal, identificados mediante análisis de regresión logística, fueron: edad gestacional al parto $(p<0,001)$, anomalía congénita $(p<0,001)$, IBA $(p=0,02)$ e hipertensión arterial $(p=0,03)$. Las principales causas de muerte perinatal fueron: hipoxia (aguda o crónica) 28\%, infección congénita 23\% (preferentemente neumonía 18\%), desprendimiento prematuro de placenta con hipoxia y shock hipovolémico $18 \%$, anomalía congénita $18 \%$ y síndrome hipertensivo con hipoxia aguda o crónica 7\%. Conclusiones: Entre las 22 y 34 semanas de gestación, el parto prematuro por IBA fue la causa más frecuente de muerte perinatal, la edad gestacional al parto fue el principal factor de riesgo de mortalidad y la hipoxia fue la causa más frecuente de muerte.

\section{PALABRAS CLAVE: Mortalidad perinatal, nacimiento prematuro, infección congénita}

\section{SUMMARY}

Aim: To determine perinatal mortality of premature birth and the risk factors associated using perinatal clinical data, laboratory results and histopathological fetal, neonatal and placental findings. Methods: Retrospective cohort study of 407 preterm single births at 22.0-34.0 weeks gestation. Fetal and neonatal deaths until 7 days of life were studied. One hundred and twenty two children died (64 fetal deaths and 58 neonatal deaths), 78 had autopsy. Results: Perinatal mortality was 30\% (122/407). Of total deaths, $71 \%(87 / 122)$ occurred before 30 weeks gestation and $81 \%(99 / 122)$ occurred in very low birth weight infants $(<1500 \mathrm{~g})$. The main causes of perinatal mortality according to associated factors for premature birth were: ascending bacterial infection ( $\mathrm{ABI})$ 41\% (50/122), congenital anomalies 20\% (24/122) and hypertension 12\% (15/122). 
Risk factors for perinatal mortality, identified by logistic regression analisis were: births at gestational age $p<0.001$, congenital anomalies $p<0.001, A B I p=0.02$ and hypertension $p=0.03$. The major causes of perinatal death were: hypoxia produced by diverse conditions (acute or chronic) $28 \%$, congenital infections $23 \%$ (mostly congenital pneumonia 18\%), abruptio placentae with hypoxia and hypovolemic shock $18 \%$, congenital anomalies $18 \%$ and hypertensive syndrome with acute or chronic hypoxia $7 \%$. Conclusions: In gestations between 22.0-34.0 weeks, premature birth caused by ABI was the most important cause of perinatal death, gestational age at birth was identified as the major risk factor for mortality and hypoxia the main cause of death.

\section{KEY WORDS: Perinatal mortality, premature birth, congenital infection}

\section{INTRODUCCIÓN}

Se estima que cada año ocurren en el mundo seis millones de muertes perinatales y el parto prematuro es su principal causa $(1,2)$.

Los nacimientos prematuros son responsables del 75 a $80 \%$ de la mortalidad perinatal, principalmente antes de las 32 semanas (2-4), de más de la cuarta parte de la morbilidad a largo plazo y de la mayoría de las complicaciones neurológicas y respiratorias que presentan los neonatos $(2,5)$.

En el Hospital Clínico San Borja Arriarán los nacimientos entre 22 y 34 semanas en el $69 \%$ provienen de partos espontáneos y en el $31 \%$ de partos por indicación médica (6). La infección bacteriana ascendente (IBA) es el factor asociado más frecuente en el parto espontáneo $(51 \%)$ y la preeclampsia $(62 \%)$ el más común en el nacimiento indicado (6).

La frecuencia de la IBA es muy alta entre las 20 y 32 semanas de gestación $(7,8)$. Es causa común de parto prematuro, $90 \%$ a las 24 semanas y $60 \%$ a las 32 semanas $(7,8)$. Representa el $33 \%$ de las muertes fetales entre 22 y 30 semanas principalmente por neumonía congénita (9).

La etiología de la muerte fetal y neonatal es posible conocerla luego de analizar los datos perinatales clínicos, los resultados de laboratorio y los hallazgos patológicos del feto, neonato y placenta (9-14).

En la mortalidad neonatal habitualmente participan condiciones que se inician en la vida intrauterina, y el neonatólogo recibe un niño gravemente enfermo con pocas posibilidades de sobrevida. La comprensión de las condiciones asociadas a la mortalidad perinatal y la prevención del parto prematuro mediante intervenciones producto del conocimiento de los factores de riesgo, parecen ser las medidas más adecuadas para reducir el nacimiento prematuro y la mortalidad asociada (15).

El objetivo de este estudio fue conocer la mortalidad perinatal del parto prematuro entre 22 y 34 semanas de gestación con embarazos únicos y los factores de riesgo asociados, usando los datos perinatales clínicos, los resultados de laboratorio y los hallazgos patológicos del feto, neonato y placenta.

\section{PACIENTES Y MÉTODOS}

Estudio retrospectivo de la mortalidad perinatal del nacimiento único prematuro entre las 22,0 y 34,0 semanas de gestación, atendido en el Servicio de Obstetricia y Ginecología del Hospital San Borja Arriarán, en el período entre el 1 de enero de 2007 y el 30 de junio de 2009.

Este trabajo analizó la muerte perinatal de serie de partos prematuros entre 22 y 34 semanas publicada recientemente (6). El Comité de Ética de la Institución aprobó la realización de este trabajo. Mortalidad perinatal y condiciones asociadas. Se estudiaron las muertes fetales y neonatales precoces (hasta los 7 días de vida). La causa asociada a la muerte fetal y neonatal se conoció luego de analizar los datos perinatales clínicos, los resultados de laboratorio y los hallazgos patológicos del feto, neonato y placenta (9-14). El estudio anatomopatológico lo realizaron dos patólogos (EK y CM). Determinar la causa de la muerte fetal o neonatal en la autopsia es con frecuencia complejo; pueden existir dos o más condiciones presentes, en esos casos se eligió la de mayor importancia asociada con la muerte perinatal. Se encontraron las siguientes causas asociadas a la muerte perinatal y su descripción diagnóstica:

a) Infecciones: Congénita: Neumonía, compromiso inflamatorio agudo alveolar, intersticial y de vía aérea de origen intrauterino, que se presenta en el feto (congénita) y/o neonato (connatal) dentro de las 24 horas de vida. En la autopsia hay signos morfológicos de hipoxia y shock, asociada con corioamnionitis y funisitis histológica agudas placentarias $(9,16,17)$. Sepsis por L. monocytogenes, compromiso séptico fetal multisistémico con microabscesos pulmonares, hepáticos, renales, subpericar- 
dicos y suprarrenales y placenta con vellositis e intervellositis aguda abscedada $(9,18)$. Sepsis por C.albicans, compromiso séptico fetal multisistémico y signos morfológicos de shock (19). Sífilis congénita por Treponema pallidum, con compromiso multisistémico fetal, hidrops no inmune, neumonía alba, fibrosis pancreática, intestinal y hepática con signos morfológicos de shock, placentomegalia y vellositis crónica linfo-plasmocitaria (9). Neonatal: Enterocolitis necrotizante (ECN) con sepsis, cuadro clínico compatible con resección intestinal, shock séptico y hallazgos específicos en la autopsia: enterocolitis necrotizante hemorrágica por un factor hipóxico-isquémico de la mucosa intestinal y signos morfológicos de shock séptico (20).

b) Desprendimiento prematuro placenta normoinserta: Separación total o parcial de la placenta del útero y con manifestaciones fetales de asfixia aguda o crónica y shock que conducen habitualmente a la muerte fetal o inmediatamente después de nacer $(9,21,22)$. La lesión placentaria más frecuentemente encontrada es la hemorragia retroplacentaria.

c) Anomalía congénita: Cromosómica, confirmada por cariograma en líquido amniótico o sangre fetal. Síndrome de Turner (XO), trisomía 18, trisomía 21 (9,23). No cromosómica, habitualmente malformaciones de un órgano o sistema $(9,23)$, de causa multifactorial, producto de factores ambientales, enfermedades maternas, agentes infecciosos, físicos, químicos, uso de medicamentos o de factores mecánicos durante el primer trimestre de la gestación. Se considera que la malformación constituye el diagnóstico de la muerte perinatal.

d) Hipertensión arterial: Embarazada con preeclampsia severa o hipertensión arterial crónica. En la autopsia del feto-neonato se encuentran signos morfológicos de hipoxia aguda y de shock, con hallazgos histopatológicos placentarios de infartos vellositarios, arteriopatía decidual, hematoma retroplacentario y maduración acelerada. $(9,11,24)$.

e) Otras condiciones que producen hipoxia aguda: Prematurez extrema, enfermedad de membrana hialina, hipoplasia e inmadurez pulmonar, hemorragia intraventricular, infección intraamniótica con funisitis, otras. Prematurez extrema, neonato con los siguientes hallazgos en la autopsia: desarrollo pulmonar en fase canalicular (16-24 semanas) y signos morfológicos de hipoxia fetal aguda intrauterina (9). Enfermedad de membrana hialina, neonato con distrés respiratorio, requerimientos de $\mathrm{O} 2$ aumentado, radiología compatible y hallazgos de membranas hialinas en bronquiolos terminales, alvéolos y atelectasias (25). Hipoplasia e inmadurez pulmonar en gestaciones menores de 24 semanas, habitualmente con rotura prematura de membra- nas de inicio precoz y desarrollo pulmonar en fase canalicular en la autopsia (26). Hemorragia intraventricular (HIV) severa, neonato con hemorragia intraventricular masiva cuadriventricular (grado IV) diagnosticadas por ultrasonografía y corroborado por la autopsia (27). Infección intraamniótica, muerte fetal cuya autopsia demuestra signos morfológicos de hipoxia fetal intrauterina, ausencia de infección de órganos y la placenta tiene corioamnionitis y funisitis aguda, sin signos de deprendimiento (9). Otras causas con hipoxia aguda. Falla cardíaca fetal. Causas no precisadas.

f) Otras condiciones con hipoxia crónica y RCIU: Patología placentaria, infección viral transplacentaria (28). Patología placentaria, embarazo sin patología materno-fetal, con muerte fetal y manifestaciones de asfixia crónica, RCIU severo a la autopsia (28) y con lesiones placentarias inespecíficas: arteriopatía fetal trombótica, trombosis intervellositaria y arterial fetal, depósito aumentado de fibrinoide perivellositario, infartos vellositarios, hematoma retroplacentario (12,21-23). Infección viral transplacentaria, infección materna (inmuno globulina M positiva), con muerte fetal, con RCIU (28), hidrops fetal no inmunológico en la autopsia y asociada con hallazgos histopatológicos placentarios inespecíficos: vellositis, intervellositis, perivellositis crónica, corioamnionitis crónica $(9,12)$.

g) No precisables: Casos en que los antecedentes clínicos, de laboratorio y anatomopatológicos no fueron suficientes para aclarar la condición asociada a la muerte perinatal (9).

Expresión de resultados. Para conocer las causas de la mortalidad perinatal y los factores de riesgo, se estudiaron en conjunto los datos perinatales clínicos, los resultados de laboratorio y los hallazgos patológicos del feto, neonato y placenta. Luego estos antecedentes se analizaron en relación con los factores asociados con el parto prematuro, con la edad gestacional al parto y con la edad materna. Los resultados se expresaron como el valor de la media \pm el error estándar de la media.

Análisis estadístico de los datos. Se usó test de chi cuadrado en comparaciones biunívocas de proporciones y test t para comparación de medias. Un valor de $p<0,05$ fue considerado significativo.

\section{RESULTADOS}

Este trabajo analizó las muertes perinatales de serie publicada recientemente, sobre los factores asociados con el parto prematuro en que se incluyeron 407 partos prematuros (6).

En el periodo estudiado hubo 168 muertes perinatales, de ellas $122(72,6 \%)$ ocurrieron entre las 
22 y 34 semanas (122/168). La mortalidad fetal fue de $52,5 \%(64 / 122)$ y la neonatal precoz de $47,5 \%$ (58/122). La mortalidad perinatal de la serie de prematuros fue de $30 \%$ (122/407). Se efectuó autopsia en el $63,9 \%(78 / 122)$ de los casos: fetal $55,1 \%$ (43/78) y neonatal precoz $44,9 \%(25 / 78)$. La tasa de mortalidad perinatal fue $13,3 / 1000$ nacidos únicos (fetal 7,0 y neonatal 6,3 ).

En la Tabla I, se presenta la mortalidad perinatal según edad gestacional y peso al nacer. La mortalidad fue mayor con menor edad gestacional y menor peso al nacer. El $71,3 \%(87 / 122)$ de las muertes ocurrieron antes de las 30 semanas y el $28,7 \%$ (35/122) entre las 30 y 34 semanas. El $81,1 \%(99 / 122)$ se presentaron en nacidos que pesaron menos de $1.500 \mathrm{~g}$ y el $18,9 \%(23 / 122)$ en aquellos que pesaron entre 1.500 y $2.500 \mathrm{~g}$.

Las tasas de muerte perinatal según factor asociado al parto prematuro se muestran en la Figura 1. Las más importantes fueron: anomalía congénita $82,8 \%$ (24/29); nacimientos por infección transplacentaria $45,5 \%(5 / 11)$ y nacimientos por IBA 34\% (50/147).

Las principales causas de muerte perinatal según el factor asociado al parto prematuro (Tabla II) fueron: IBA 41,0\% (50/122): 15 muertes fetales, 25 neonatales el primer día y 10 en días siguientes; anomalía congénita 19,7\% (24/122): 17 fetales y 7 neonatales luego de nacer; hipertensión arterial
12,3\% (15/122): 8 fetales, 5 neonatales en el primer día y 2 en días siguientes.

Se analizaron los factores de riesgo asociados a la muerte perinatal (variable dependiente) identificados mediante análisis de regresión logística. Al modelo estadístico se introdujeron las variables, edad materna, edad gestacional al parto y factor asociado al parto prematuro. Los factores de riesgo asociados fueron: edad gestacional al parto $(p<0,001)$, anomalía congénita $(p<0,001)$, IBA $(p=0,02)$ e hipertensión arterial $(p=0,03)$. No hubo asociación estadísticamente significativa con patología placentaria, enfermedades médicas maternas, infección transplacentaria, causa no precisable y edad materna.

En la Tabla III se presentan las causas asociadas a la muerte perinatal. Las causas más frecuentes fueron:

a) Otras condiciones que producen hipoxia en el $28,2 \%$ (22/78, fetales 8, neonatales 14$)$; hipoxia aguda 17 casos [prematurez extrema (7 casos) especialmente nacidos por IBA, enfermedad de membrana hialina (3 casos), infección intraamniótica con funisitis (2 casos), HIV masiva grado IV (1 caso), hipoplasia pulmonar (1 caso), otras situaciones (3 casos)]; hipoxia crónica con RCIU <percentil 5 , cinco casos [nacimientos asociados con patología vascular placentaria (3 casos) e infección viral transplacentaria (2 casos)].

Tabla I

MORTALIDAD PERINATAL EN EL PARTO PREMATURO ENTRE 22-34 SEMANAS SEGÚN EDAD GESTACIONAL Y PESO AL NACER. HOSPITAL SAN BORJA ARRIARÁN 2007-2009

\begin{tabular}{|c|c|c|c|}
\hline & $\begin{array}{l}\text { Mortalidad } \\
\text { Fetal }(n: 64)\end{array}$ & $\begin{array}{r}\text { Mortalidad } \\
\text { Neonatal precoz }(\mathrm{n}: 58)\end{array}$ & $\begin{array}{r}\text { Mortalidad } \\
\text { Perinatal (n:122) }\end{array}$ \\
\hline \multicolumn{4}{|c|}{$\begin{array}{l}\text { Edad gestacional parto prematuro } \\
\text { (semanas) }\end{array}$} \\
\hline Media (EE) & $28,2(1,09)$ & $28,6(1,0)$ & $28,4(0,7)$ \\
\hline Rango 22-25.6 & $19 / 52(36,5 \%)$ & $24 / 52(46,2 \%)$ & $43 / 52(82,7 \%)$ \\
\hline $26-29.6$ & $22 / 104(21,2 \%)$ & $22 / 104(21,2 \%)$ & $44 / 104(42,3 \%)$ \\
\hline $30-34.0$ & $23 / 251(9,1 \%)$ & $12 / 251(4,8 \%)$ & $35 / 251(13,9 \%)$ \\
\hline \multicolumn{4}{|l|}{ Peso RN (gramos) } \\
\hline Media (EE) & $1058,2(68,7)$ & $1032,6(60,4)$ & $1046,0(45,9)$ \\
\hline Rango & $430-2460$ & $498-2460$ & $430-2460$ \\
\hline$<$ de 750 & $25 / 50(50,0 \%)$ & $18 / 50(36,0 \%)$ & $43 / 50(86,0 \%)$ \\
\hline $750-999$ & $14 / 48(29,2 \%)$ & $17 / 48(35,4 \%)$ & $31 / 48(64,6 \%)$ \\
\hline $1000-1499$ & $12 / 110(10,9 \%)$ & $13 / 110(11,8 \%)$ & $25 / 110(22,7 \%)$ \\
\hline $1500-1999$ & $7 / 130(5,4 \%)$ & $7 / 130(5,4 \%)$ & $14 / 130(10,8 \%)$ \\
\hline$>$ de 2000 & $6 / 69(8,7 \%)$ & $3 / 69(4,3 \%)$ & $9 / 69(13,0 \%)$ \\
\hline
\end{tabular}

EE: Error estándar 


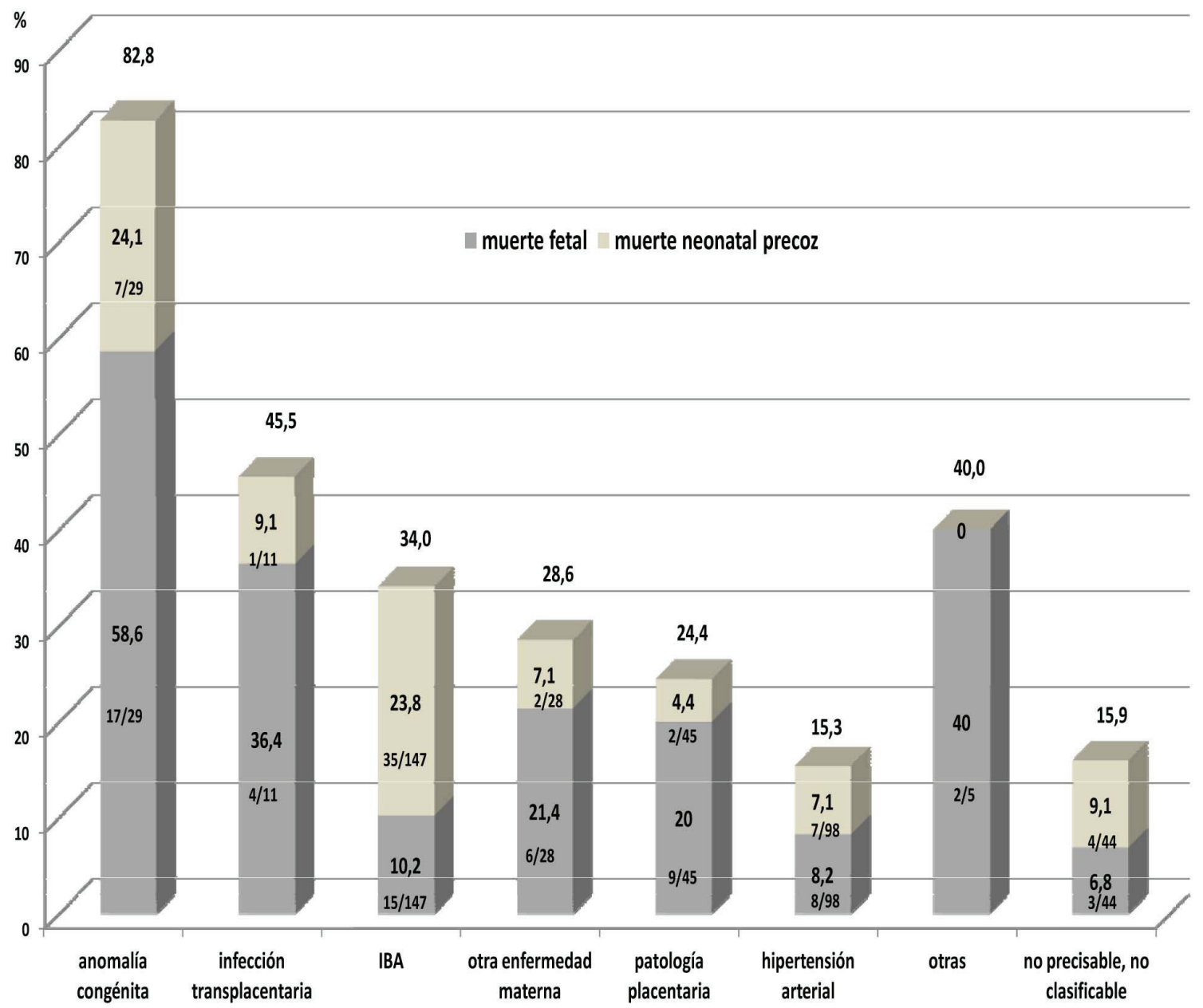

Figura 1. Tasas de muertes perinatales según factor asociado al parto prematuro entre 22-34 semanas. Hospital San Borja Arriarán 2007-2009.

Tabla II

PRINCIPALES CAUSAS DE MUERTE PERINATAL SEGÚN EL FACTOR ASOCIADO CON EL PARTO PREMATURO ENTRE 22 Y 34 SEMANAS. HOSPITAL SAN BORJA ARRIARÁN 2007-2009

\begin{tabular}{lccc}
\hline $\begin{array}{l}\text { Factores asociados con } \\
\text { el parto prematuro }\end{array}$ & $\begin{array}{c}\text { Muerte fetal } \\
(\mathrm{a}) \\
\mathrm{n:} 64\end{array}$ & $\begin{array}{c}\text { Muerte neonatal precoz } \\
(\mathrm{b}) \\
\mathrm{n}: 58\end{array}$ & $\begin{array}{c}\text { Muerte perinatal } \\
(\mathrm{a}+\mathrm{b}) \\
\mathrm{n}: 122\end{array}$ \\
\hline IBA & $15(23,4 \%)$ & $35(60,3 \%)$ & $50(41,0 \%)$ \\
Anomalía congénita & $17(26,6 \%)$ & $7(12,1 \%)$ & $24(19,7 \%)$ \\
Hipertensión arterial & $8(12,5 \%)$ & $7(12,1 \%)$ & $15(12,3 \%)$ \\
Patología placentaria & $9(14,1 \%)$ & $2(3,4 \%)$ & $11(9,0 \%)$ \\
Otra enfermedad médica materna & $6(9,4 \%)$ & $2(3,4 \%)$ & $8(6,6 \%)$ \\
Infección transplacentaria & $4(6,3 \%)$ & $1(1,7 \%)$ & $5(4,1 \%)$ \\
Otras & $2(3,2 \%)$ & 0 & $2(1,6 \%)$ \\
No precisable, no clasificable & $3(4,7 \%)$ & $4(6,9 \%)$ & $7(5,7 \%)$ \\
\hline
\end{tabular}

IBA: Infección bacteriana ascendente 
Tabla III

CAUSAS DE MUERTE PERINATAL EN EL PARTO PREMATURO ENTRE 22 Y 34 SEMANAS. HOSPITAL SAN BORJA ARRIARÁN 2007-2009

Otra infección

DPPNI con hipoxia aguda (n: 12) o crónica (n: 2)

Otra causa con hipoxia aguda

Otra causa con hipoxia crónica y RCIU severo

Preeclampsia con hipoxia aguda

Hipertensión arterial crónica con hipoxia crónica y RCIU severo

Anomalía congénita

DPPNI: Desprendimiento prematuro de placenta normo inserta. RCIU: Restricción del crecimiento intrauterino

b) Infección en el 23,0\% (18/78): neumonía congénita 14 (fetal 5 y connatal 9); sepsis congénita (L. monocytogenes, C. albicans) (2 casos); sífilis congénita (1 caso); ECN neonatal con shock séptico (1 caso).

c) $D P P N I$ con hipoxia y shock hipovolémico en el $17,9 \%$ (14/78, fetal 12, neonatal 2), con hipoxia aguda (12 casos) y con hipoxia crónica (2 casos), desprendimientos placentarios presentes en: partos prematuros por IBA (7 casos), patología placentaria primaria (2 casos), preeclampsia (2 casos), trombofilia ( 2 casos) y consumo de drogas (1 caso). d) Anomalía congénita en el 17,9\% (14/78) no cromosómica (10 casos) y cromosómica (4 casos); fetal (9 casos), neonatal (5 casos).

e) Síndrome hipertensivo con hipoxia en el 6,6\% (6/78); preeclampsia con hipoxia aguda (3 casos) e hipertensión crónica con hipoxia crónica (3 casos); fetal (4 casos) y neonatal ( 2 casos).

f) No precisable en el 5,1\% (4/78).

\section{DISCUSIÓN}

Este trabajo revela que la mortalidad perinatal en el parto prematuro entre 22 y 34 semanas con embarazos únicos en un hospital público de Santiago fue de $30 \%$ y correspondió al $74 \%$ de todas las muertes perinatales ocurridas en el período del estudio. La edad gestacional al parto, la IBA, la anomalía congénita y la hipertensión arterial se identificaron como factores de riesgo de muerte perinatal mediante análisis de regresión logística.

Estos resultados sugieren que para reducir la muerte que ocurre en el momento del parto, nece- sariamente deben introducirse intervenciones que prevengan el parto prematuro y que eviten el nacimiento de niños de muy bajo peso.

El parto prematuro causado por IBA, habitualmente espontáneo, fue el que produjo mayor muerte perinatal en esta serie $(41 \%)$. Los niños fallecieron por neumonía congénita (fetal o connatal dentro de las primeras 24 horas de vida) (9), por hipoxia aguda secundaria a DPPNI o por prematurez extrema. El neonatólogo poco pudo hacer con un niño que nació muerto o gravemente enfermo. Frente a estos resultados y porque estos daños pueden ser prevenibles, es muy conveniente elaborar estrategias obstétricas eficientes tendientes a reducir el parto prematuro y la mortalidad asociada por esta causa: pesquisa y tratamiento en la población de riesgo de las vaginitis $(6,29)$ especialmente por Streptococcus agalactiae $(6,30)$ y de vaginosis bacteriana desde el $2^{\circ}$ trimestre del embarazo; pesquisa y tratamiento de las infecciones urinarias (6); iniciar y evaluar protocolos sobre el uso de antibióticos en situaciones en que se suele encontrar infección intraamniótica (sangrado vaginal-desprendimiento amniocorial, cérvix corto) (31-33); uso de progesterona (34) y de cerclaje (35) preferentemente profiláctico sin esperar acortamiento cervical (36).

El niño malformado (muere el $83 \%$ entre 22 y 34 semanas) fue responsable del $20 \%$ de las muertes perinatales. La muerte ocurrió por hipoxia aguda, patología asociada a la malformación. Las muertes fueron fetales o inmediatamente después del nacimiento. La malformación fetal del tubo neural puede reducirse con uso de ácido fólico pregestacional (37), pero la mortalidad por esta causa es difícil de 
disminuir por la gravedad de las malformaciones y porque la legislación vigente no permite el aborto en estos casos.

La hipertensión arterial fue responsable del $12 \%$ de la mortalidad perinatal. Las muertes ocurrieron por hipoxia aguda en la preeclampsia severa, por hipoxia crónica con RCIU severo en la hipertensión crónica y por DPPNI. Casi la totalidad de estas muertes son fetales o en el primer día de vida. El manejo de estos neonatos en cuidados intensivos es complejo y con pobres resultados. La mortalidad puede reducirse previniendo la preeclampsia posiblemente con el uso de aspirina en bajas dosis, $\mathrm{L}$ arginina, antioxidantes $(38,39)$ y seleccionando adecuadamente el momento de la interrupción del embarazo.

La limitación de este estudio fue la exclusión de 44 casos sin autopsia fetal-neonatal. Pero este error en la selección de pacientes no debió modificar los resultados de este estudio, porque la decisión de no hacer autopsia fue por determinación de los padres y ocurrió al azar. Además, los datos clínicos y de laboratorio de los casos excluidos permiten sugerir que la distribución de los posibles factores de muerte perinatal asociados en este grupo no fue diferente del encontrado en el grupo con autopsia.

\section{CONCLUSIÓN}

El uso de datos clínicos maternos y perinatales, resultados de laboratorio y hallazgos patológicos del feto, neonato y placenta resultaron muy útiles para conocer tanto los factores asociados con el parto prematuro como también los factores de riesgo de mortalidad perinatal. El conocimiento de estos factores permite que las intervenciones tendientes a reducir el parto prematuro y la mortalidad asociada sean más eficientes.

\section{REFERENCIAS}

1. Matendo RM, Engmann CM, Ditekemena JD, Gado J, Tshefu A, McClure EM, et al. Challenge of reducing perinatal mortality in rural Congo: findings of a prospective, population-based study. J Health Popul Nutr 2011;5:532-40.

2. Goldenberg RL, Culhane JF, lams JD, Romero R. Epidemiology and causes of preterm birth. Lancet 2008;371:75-84.

3. McCormick MC. The contribution of low birth weight to infant mortality and childhood morbidity. N Engl J Med 1985;312: 82-90.

4. Ticona M, Huanco D. Mortalidad perinatal hospitalaria en el Perú: Factores de riesgo. Rev Chil Obstet Ginecol 2005;70:313-7.

5. Saigal S, Doyle LW. Preterm Birth 3. An overview of mortality and sequelae of preterm birth from infancy to adulthood. Lancet 2008;371:261-9.

6. Ovalle A, Kakarieka E, Rencoret G, Fuentes A, del Río MJ, Morong $\mathrm{C}$, et al. Factores asociados con el parto prematuro entre 22 y 34 semanas en un hospital público de Santiago. Rev Med Chile 2012;140:19-29.

7. Sebire NJ, Goldin RD, Regan L. Histological chorioamnionitis in relation toclinical presentation at 14-40 weeks of gestation. J Obstet Gynaecol 2001;21:242-5.

8. Al-Adnani M, Sebire NJ. The role of perinatal pathological examination in subclinical infection in obstetrics. Best Pract Res Clin Obstet Gynaecol 2007;21:505-21.

9. Ovalle A, Kakarieka E, Correa A, Vial MT, Aspillaga C. Estudio anátomo-clínico de las causas de muerte fetal. Rev Chil Obstet Ginecol 2005;70:303-12.

10. Corabian P, Scott NA, Lane C, Guyon G. Guidelines for investigating stillbirths: an update of a systematic review. J Obstet Gynaecol Can 2007;29:560-7.

11. Arias F, Victoria A, Cho K, Kraus F. Placental histology and clinical characteristics of patients with preterm premature rupture of membranes. Obstet Gynecol 1997;89: 265-71.

12. Redline RW. Placental Pathology: A systematic approach with clinical correlations. Placenta 2008;29 Supple A:S86-91.

13. Ozkara E, Canturk G, Canturk N, Ozata AB, Yavuz MF. Evaluation of forensic perinatal and neonatal autopsies in Istanbul. Indian J Pediatr 2009;76:167-70.

14. Pakis I, Karapirli M, Karayel F, Turan A, Akyildiz E, Polat O. Quality assessment of perinatal and infant postmortem examinations in Turkey. J Forensic Sci 2008;53:1166-8.

15. lams JD, Romero R, Culhane JF, Goldenberg RL. Primary, secondary, and tertiary interventions to reduce the morbidity and mortality of preterm birth. Lancet 2008;371:164-75.

16. Faix RG. Neumonía congénita. Medscape. 29.03.2011.

17. De Paepe ME, Friedman RM, Gundogan F, Pinar H, Oyer CE. The histologic fetoplacental inflammatory response in fatal perinatal group B-streptococcus infection. J Perinatol 2004;24:441-5.

18. Larraín D, Abarzúa $F$, Jourdan F, Merino P, Belmar C, García P. Listeria monocytogenes infection in pregnancy: experience of Pontificia Universidad Católica de Chile University Hospital. Rev Chilena Infectol 2008;5:336-41.

19. Leroy O, Gangneux JP, Montravers P, Mira JP, Gouin $\mathrm{F}$, Sollet JP, et al. Epidemiology, management, and risk factors for death of invasive Candida infections in critical care: a multicenter, prospective, observational study in France (2005-2006). Crit Care Med 2009;37:1612-8.

20. Polin RA. Management of neonates with suspected or proven early-onset bacterial sepsis. Pediatrics 2012;129:1006-15.

21. Perrin VDK. Pathology of the placenta. Chapter: Placenta as a reflection of maternal disease. p 57-70. New York, Churchill Livingstone Inc; 1984.

22. Fox H. Pathology of the placenta. Chapter: Histological abnormalities of the placenta. p 149-197. Philadelphia, Pa: WB Saunders Co; 1978. 
23. Keeling JW. Fetal and Neonatal Pathology. SprigerVerlag. Berlin Heidelgerg; 1987.

24. Kovo M, Schreiber L, Ben-Haroush A, Wand S, GoIan A,Bar J. Placental vascular lesion differences in pregnancyinduced hypertension and normotensive fetal growth restriction. Am J Obstet Gynecol 2010;202:561.e1-5.

25. Fidanovski D, Milev V, Sajkovski A, Hristovski A, Sofijanova A, Kojić L, et al. Mortality risk factors in premature infants with respiratory distress syndrome treated by mechanical ventilation. Srp Arh Celok Lek 2005;133:29-35.

26. Williams O, Hutchings G, Debieve F, Debauche C. Contemporary neonatal outcome following rupture of membranes prior to 25 weeks with prolonged oligohydramnios. Early Hum Dev 2009;85:273-7.

27. Sarkar S, Bhagat I, Dechert R, Schumacher RE, Donn $\mathrm{SM}$. Severe intraventricular hemorrhage in preterm infants: comparison of risk factors and short-term neonatal morbidities between grade 3 and grade 4 intraventricular hemorrhage. Am J Perinatol 2009;26:419-24.

28. Milad M, Novoa JM, Fabres J, Samamé MM, Aspillaga C. Recomendación sobre curvas de crecimiento intrauterino. Rev Chil Pediatr 2010; 81:264-74.

29. Lamont RF, Nhan-Chang CL, Sobel JD, Workowski K, Conde-Agudelo A, Romero R. Treatment of abnormal vaginal flora in early pregnancy with clindamycin for the prevention of spontaneous preterm birth: a systematic review and metaanalysis. Am J Obstet Gynecol 2011;205:177-90.

30. Håkansson S, Källén K. Impact and risk factors for early-onset group B streptococcal morbidity: analysis of a national, population-based cohort in Sweden 1997-2001. BJOG 2006;113:1452-8.

31. Gómez R, Romero R, Nien JK, Medina L, Carstens M, Kim YM, et al. Idiopathic vaginal bleeding during pregnancy as the only clinical manifestation of intrauterine infection. J Matern Fetal Neonatal Med 2005;18:31-7.

32. Madan I, Romero R, Kusanovic JP, Mittal P, Chaiworapongsa $T$, Dong $Z$, et al. The frequency and clinical significance of intra-amniotic infection and/or inflammation in women with placenta previa and vaginal bleeding: an unexpected observation. J Perinat Med 2010;38:275-9.

33. Hassan S, Romero R, Hendler I, Gómez R, Khalek N, Espinoza J, et al. A sonographic short cervix as the only clinical manifestation of intra-amniotic infection. $J$ Perinat Med 2006;34:13-9.

34. Romero R, Nicolaides K, Conde-Agudelo A, Tabor A, O'Brien JM, Cetingoz E, et al. Vaginal progesterone in women with an asymptomatic sonographic short cervix in the midtrimester decreases preterm delivery and neonatal morbidity: a systematic review and metaanalysis of individual patient data. Am J Obstet Gynecol 2012;206:124.e1-19.

35. Berghella V, Rafael TJ, Szychowski JM, Rust OA, Owen J. Cerclage for short cervix on ultrasonography in women with singleton gestations and previous preterm birth. Obstet Gynecol 2011;117:663-71.

36. Ovalle A, Valderrama O, Rencoret G, Fuentes A, del Río MJ, Kakarieka E, et al. Cerclaje profiláctico en mujeres con nacimientos prematuros espontáneos previos asociados con infección bacteriana ascendente. Rev Chil Obstet Ginecol 2012;77:98-105.

37. Barboza Argüello ML, Umaña Solís LM. Impact of the fortification of food with folic acid on neural tube defects in Costa Rica. Rev Panam Salud Pública 2011;30:1-6.

38. Vadillo-Ortega F, Perichart-Perera O, Espino S, AvilaVergara MA, Ibarra I, Ahued R, et al. Effect of supplementation during pregnancy with L-arginine and antioxidant vitamins in medical food on pre-eclampsia in high risk population: randomized controlled trial. BMJ 2011;342:d2901.

39. Roberge $S$, Giguère $Y$, Villa $P$, Nicolaides $K$, Vainio $M$, Forest JC, et al. Early Administration of low-dose aspirin for the prevention of severe and mild preeclampsia: a systematic review and meta-analysis. Fetal Diagn Ther 2012;31:141-6. 